\title{
Sound Attenuation at High Temperatures in Pt
}

\author{
R.K. SingH* AND K.K. PANDEY \\ H.C.P.G. College, Varanasi-221001, U.P., India
}

(Received October 4, 2005)

\begin{abstract}
Ultrasonic attenuation due to phonon-phonon interaction and thermoelastic loss was evaluated in VIII group transition metal $\mathrm{Pt}$ in a wide temperature range $(100 \mathrm{~K}$ to $1500 \mathrm{~K})$ for longitudinal and shear waves along $\langle 100\rangle,\langle 110\rangle$ and $\langle 111\rangle$ directions and for shear waves polarised along different directions. Electrostatic and Born-Mayer potentials were used to obtain second and third order elastic constants, taking nearest-neighbour distance and hardness parameter as input data. Second and third order elastic constants data obtained at different temperatures were used to obtain Gruneisen parameters and non-linearity or anisotropy parameters which in turn were used to evaluate $\left(\frac{\alpha}{f^{2}}\right)_{l}$ and $\left(\frac{\alpha}{f^{2}}\right)_{s}$ in Akhiezer regime. The results were discussed and it was found that at lower temperatures $\left(\frac{\alpha}{f^{2}}\right)$ increases rapidly with temperature and then rate of increase becomes very small. Contribution to the total attenuation due to thermoelastic loss is negligible so that due to phonon-phonon interaction establishing that major part of energy from sound wave is removed due to interaction with thermal phonons (lattice vibrations).
\end{abstract}

PACS numbers: 62.20.Dc, 62.65.+k

\section{Introduction}

Platinum $(Z=78)$ is a silvery white transition metal of VIII group in VI period with an outer electronic configuration of $5 d^{9} 6 s^{1}$ and shows high catalytic activity. It is highly malleable and ductile metal with high melting point $(2041.4 \mathrm{~K})$. Platinum is corrosion resistant and its wear and tarnish resistance characteristics are well suited for making fine jewelry. Other distinctive properties include resistance to chemical attack, excellent high temperature characteristics and sta-

\footnotetext{
${ }^{*}$ corresponding author; e-mail: rksingh_17@rediffmail.com. Present address: Physics Department, B.H.U., Varanasi-221005, India.
} 
ble electrical properties. All these properties have been exploited for industrial applications.

In recent past, elastic [1], thermal and mechanical [2] properties of Pt have been investigated extensively at room temperature as well as in wide temperature region (up to $1500 \mathrm{~K}$ ). So far as sound characterisation of platinum is concerned, no result is available for this metal in wide temperature range. Ultrasonic attenuation studies have been made in different types of solids viz. dielectric [3], mixed dielectric [4], semiconducting $[5,6]$ and metallic $[7,8]$ substances at room temperature as well as at higher temperatures. But results are available up to $\approx 500 \mathrm{~K}$. Due to high melting point of platinum and its important high temperature uses, ultrasonic attenuation study has been made for Pt from $100 \mathrm{~K}$ to $1500 \mathrm{~K}$.

Different causes may be attributed to the attenuation of ultrasonic waves propagating through solids (crystalline or amorphous). Of these important factors for sound attenuation are electron-phonon interaction, phonon-phonon ( $\mathrm{p}-\mathrm{p})$ interaction, thermoelastic loss and loss due to screw and edge dislocations. Phononphonon interaction is the principal cause of ultrasonic attenuation at high temperatures viz. $100 \mathrm{~K}$ and above in all types of solids i.e. metallic, semiconducting and dielectric [4-8]. In metals a small contribution (about 2 to $5 \%$ ) of total attenuation coefficient occurs due to thermoelastic loss i.e. due to heat transfer from compressed to rarefied parts of metals due to strain caused by applied sound waves.

Sound attenuation studies offer the possibility to detect and characterize microstructural properties as well as flaws in materials, controlling material behaviours, based on physical mechanisms to predict future performance of the material. Structural inhomogeneities, elastic parameters, non-linearity parameters are well connected with frequency or temperature dependence of ultrasonic attenuation mechanisms. Starting from electrostatic and Born-Mayer repulsive potentials and taking nearest-neighbour distance and Born repulsive parameters as input data and considering interactions up to 2nd nearest neighbours, second and third order elastic constants have been obtained at different temperatures which in turn have been used to obtain non-linearity coupling parameters and hence ultrasonic absorption coefficients.

The present communication deals with temperature variation of ultrasonic attenuation due to $\mathrm{p}-\mathrm{p}$ interaction from $100 \mathrm{~K}$ to $1500 \mathrm{~K}$ along $\langle 100\rangle,\langle 110\rangle$ and $\langle 111\rangle$ crystallographic directions of propagation for longitudinal and shear waves and for shear waves polarized along different directions. Ultrasonic attenuation due to thermoelastic loss has been evaluated and it has been found that its contribution relative to contribution made by $\mathrm{p}-\mathrm{p}$ interaction is about $2-5 \%$. Temperature variation of ultrasonic attenuation has been found to increase rapidly at lower temperatures and afterwards rate of increase becomes small which nearly saturates beyond $1000 \mathrm{~K}$ along all the directions of propagation for longitudinal and shear waves. 


\section{Theory}

\subsection{Theory of second and third order elastic constants}

Second and third order elastic constants (SOEC and TOEC), $C_{i j}^{0}$ and $C_{i j k}^{0}$, at $0 \mathrm{~K}$ have been obtained using electrostatic and Born-Mayer potentials and following Brugger's [9] definition of elastic constants. Repulsive parameter and nearest-neighbour distance have been used as input data and interaction up to next nearest-neighbours has been considered. According to Brugger's definition, $n$-th order elastic constant is defined as

$$
C_{i j k l m n \ldots}=\left(\partial u / \partial \varepsilon_{i j} \partial \varepsilon_{k l} \partial \varepsilon_{m n} \ldots\right) \text {, }
$$

where $u$ is the crystal free-energy density and $\varepsilon_{i j}$ is strain tensor. In Voigt notation $C_{I J K}$ replaces $C_{i j k l m n}$ in which $i j \rightarrow I \ldots$ etc. For cubic crystals (Pt has fcc structure) three independent $\operatorname{SOEC}\left(C_{11}, C_{12}\right.$, and $\left.C_{44}\right)$ and six independent TOEC $\left(C_{111}, C_{112}, C_{144}, C_{166}, C_{456}\right.$, and $\left.C_{123}\right)$ occur. Using the theory discussed in [9], SOEC and TOEC at $0 \mathrm{~K}$ viz. $C_{i j}^{0}$ and $C_{i j k}^{0}$ obtained are given as

$$
\begin{aligned}
& C_{11}^{0}=-1.56933 \frac{z^{2} e^{2}}{r_{0}^{4}}+\frac{1}{q r_{0}}\left(\frac{1}{r_{0}}+\frac{1}{q}\right) Q\left(r_{1}\right)+\frac{2}{q r_{0}}\left(\frac{1}{\sqrt{2} r_{0}}+\frac{1}{q}\right) Q\left(r_{2}\right), \\
& C_{12}^{0}=C_{44}^{0}=0.344778 \frac{z^{2} e^{2}}{r_{0}^{4}}+\frac{1}{q r_{0}}\left(\frac{1}{\sqrt{2} r_{0}}+\frac{1}{q}\right) Q\left(r_{2}\right), \\
& C_{111}^{0}=10.26390 \frac{z^{2} e^{2}}{r_{0}^{4}}-\left(\frac{3}{q r_{0}^{2}}+\frac{3}{q^{2} r_{0}}+\frac{1}{q^{3}}\right) Q\left(r_{1}\right) \\
&-\left(\frac{3}{\sqrt{2} r_{0}^{2} q}+\frac{3}{q^{2} r_{0}}+\frac{\sqrt{2}}{q^{3}}\right) Q\left(r_{2}\right), \\
& C_{112}^{0}=C_{166}^{0}=1.209625 \frac{z^{2} e^{2}}{r_{0}^{4}}+\left(\frac{3}{2 \sqrt{2} r_{0}^{2} q}+\frac{3}{q^{2} r_{0}}+\frac{\sqrt{2}}{q^{3}}\right) Q\left(r_{2}\right), \\
& C_{123}^{0}= C_{144}^{0}=C_{456}^{0}=1.20862 \frac{z^{2} e^{2}}{r_{0}^{4}}-\left(\frac{3}{2 \sqrt{2} r_{0}^{2} q}+\frac{3}{2 q^{2} r_{0}}+\frac{1}{\sqrt{2} q^{3}}\right) Q\left(r_{2}\right), \\
& Q\left(r_{1}\right)=A \exp \left(-\frac{r_{0}}{q}\right), \quad Q\left(r_{2}\right)=A \exp \left(-\frac{r_{0} \sqrt{2}}{q}\right) \cdot
\end{aligned}
$$

According to lattice dynamics developed by Leibfried and Ludwig [10] temperature variation of SOEC and TOEC have been obtained by adding vibrational contribution to elastic constants.

SOEC and TOEC at any temperature are given by

$$
C_{i j}(T)=C_{i j}^{0}+C_{i j}^{\mathrm{vib}}
$$

and 


$$
C_{i j k}(T)=C_{i j k}^{0}+C_{i j k}^{\mathrm{vib}}
$$

where $C_{i j}^{\text {vib }}$ and $C_{i j k}^{\text {vib }}$ are vibrational contributions to elastic constants where

$$
\begin{aligned}
& C_{11}^{\mathrm{vib}}=f^{1,1} G_{1}^{2}+f^{2} G_{2}, \quad C_{12}^{\mathrm{vib}}=f^{1,1} G_{1}^{2}+f^{2} G_{1,1}, \quad C_{44}^{\mathrm{vib}}=f^{2} G_{1,1}, \\
& C_{111}^{\mathrm{vib}}=f^{1,1,1} G_{1}^{3}+3 f^{2,1} G_{2} G_{1}+f^{3} G_{3}, \\
& C_{112}^{\mathrm{vib}}=f^{1,1,1} G_{1}^{3}+f^{2,1}\left(2 G_{1,1}+G_{2}\right) G_{1}+f^{3} G_{2,1}, \\
& C_{123}^{\mathrm{vib}}=f^{1,1,1} G_{1}^{3}+3 f^{2,1} G_{1} G_{1,1}+f^{3} G_{1,1,1}, \\
& C_{144}^{\mathrm{vib}}=f^{2,1} G_{1} G_{1,1}+f^{3} G_{1,1,1}, \\
& C_{456}^{\mathrm{vib}}=f^{3} G_{1,1}, \quad C_{166}^{\mathrm{vib}}=f^{2,1} G_{1} G_{1,1}+f^{3} G_{2,1},
\end{aligned}
$$

where

$$
\begin{aligned}
& G_{3}=2 Q\left(r_{1}\right)\left(30+30 q_{0}+9 q_{0}^{2}-q_{0}^{3}-q_{0}^{4}\right) H+2 G_{2.1}, \\
& G_{2,1}=Q\left(r_{2}\right)\left(15 \sqrt{2}+15 q_{0}+\frac{9}{\sqrt{2} q_{0}^{2}}-q_{0}^{3}-\sqrt{2} q_{0}^{4}\right) H, \\
& G_{2}=2 Q\left(r_{0}\right)\left(-6-6 q_{0}-q_{0}^{2}+q_{0}^{3}\right) H+2 G_{1.1}, \\
& G_{1,1}=Q\left(r_{2}\right)\left(-3 \sqrt{2}-6 \sqrt{2} q_{0}-\sqrt{2} q_{0}^{2}+2 q_{0}^{3}\right) H, \\
& G_{1}=2 Q\left(r_{0}\right)\left(2+2 q_{0}-q_{0}^{2}\right)+Q\left(r_{2}\right)\left(\sqrt{2}+2 q_{0}-\sqrt{2} q_{0}^{2}\right) H,
\end{aligned}
$$

where

$$
\begin{aligned}
& Q\left(r_{1}\right)=A \exp \left(-\frac{r_{0}}{q}\right) \quad \text { and } Q\left(r_{2}\right)=A \exp \left(-\frac{r_{0} \sqrt{2}}{q}\right), \\
& f^{2}=f^{3}=\frac{\eta w_{0}}{8 r_{0}^{3}} \operatorname{coth} X \\
& f^{1,1}=f^{2,1}=-\frac{1}{2 r_{0}^{3}} \frac{\eta w_{0}}{48} \frac{X}{\sinh ^{2} X}+\operatorname{coth} X \\
& f^{1,1,1}=\frac{\eta w_{0}}{384 r_{0}^{3}}\left[\left(\frac{\eta w_{0}}{k T}\right)^{2} \frac{\operatorname{coth} X}{6 \sinh ^{2} X}+\frac{X}{\sinh ^{2} X}+\operatorname{coth} X\right] \\
& X=\frac{\eta w_{0}}{2 k T}, \quad \eta=\frac{h}{2 \pi} \quad \text { and } \quad k \quad \text { is Boltzmann constant, } \\
& H=\left[\left(\frac{r_{0}}{q-2}\right) Q\left(r_{1}\right)+2\left(\frac{r_{0}}{q}-\sqrt{2}\right) Q\left(r_{2}\right)\right]^{-1} .
\end{aligned}
$$




\subsection{Theory of sound attenuation}

In the Akhiezer regime a sound wave passing through a solid can be attenuated by two processes [11]. First, if the wave is longitudinal, periodic contractions and dilations in the solid induce a temperature wave via thermal expansion. Energy is dissipated by heat conduction between regions of different temperatures. This is called thermoelastic loss. Second, dissipation occurs as the gas of thermal phonons tries to reach an equilibrium characterized by a local (sound wave induced) strain. This is an internal friction mechanism.

The physical basis for obtaining attenuation coefficient is that the elastic constants contributed by the thermal phonons relax [12]. The phonon contribution to the unrelaxed elastic constants is evaluated by taking into consideration the change in energy of the thermal phonons due to applied instantaneous strain. The frequency of each mode $\nu_{i}$ is changed by $\frac{\partial \nu_{i}}{\nu_{i}}=-\gamma_{i}^{j} S_{j}$, where $\gamma_{i}^{j}$ is generalised Gruneisen parameter and $S_{j}$ is instantaneous strain. It is assumed that all the phonons of a given direction of propagation and polarization have equal change in frequency. Then phonons of $i$-th branch and $j$-th mode suffer a change in temperature $\frac{\Delta T_{i}}{T_{0}}=-\gamma_{i}^{j} S_{j}$ ( $T$ is the temperature). A relaxed elastic constant is obtained after there is phonon-phonon coupling among various branches and the $\Delta T_{i}$ relax to a common temperature change, $\Delta T$, given by $\frac{\Delta T}{T}=-\left\langle\gamma_{i}^{j}\right\rangle S_{j}$, where $\left\langle\gamma_{i}^{j}\right\rangle$ is the average value of $\gamma_{i}^{j}$. Thermal relaxation time is

$$
\tau=\tau_{s}=\frac{\tau_{l}}{2}=\frac{3 K}{C_{V}\langle v\rangle^{2}}
$$

where $K$ is thermal conductivity, $C_{V}$ is specific heat per unit volume and $\langle v\rangle$ is Debye average velocity.

According to Mason and Batemann [12], SOEC and TOEC (which are measure of anharmonicity of the crystal) are related by Gruneisen parameter $\gamma_{i}^{j}$ and hence by non-linearity parameter, $D$. Ultrasonic attenuation due to phononphonon interaction in Akhiezer regime $(\omega \tau \ll 1)[12]$ is given by

$$
\left(\frac{\alpha}{f^{2}}\right)_{\mathrm{p}-\mathrm{p}}=\frac{2 \pi^{2} D E_{0} \tau}{3 v^{3}},
$$

where non-linearity coupling constant

$$
D=9\left\langle\left(\gamma_{i}^{j}\right)^{2}\right\rangle-\frac{3\left\langle\gamma_{i}^{j}\right\rangle^{2} C_{V} T}{E_{0}}
$$

$\left\langle\left(\gamma_{i}^{j}\right)^{2}\right\rangle$ and $\left\langle\gamma_{i}^{j}\right\rangle$ are square average and average square Gruneisen parameters, $v$ is sound wave velocity for longitudinal waves $\left(v_{l}\right)$ and for shear waves $\left(v_{s}\right)$ and $d$ is density.

Debye average velocity is given by

$$
\frac{3}{\langle v\rangle^{3}}=\frac{1}{v_{l}^{3}}+\frac{2}{v_{s}^{3}} \text {. }
$$

Propagation of sound wave through crystal produces compression and rarefactions as a result heat is transmitted from compressed region (at higher 
temperature) to rarefied region (at lower temperature) and hence thermoelastic loss occurs, which is given by [13]

$$
\left(\frac{\alpha}{f^{2}}\right)_{\mathrm{th}}=\frac{4 \pi^{2}\left\langle\gamma_{i}^{j}\right\rangle^{2} K T}{2 d v_{l}^{5}}
$$

\section{Results and discussions}

Using the theory discussed above values of SOEC and TOEC obtained at absolute zero temperature are given in Table I. Thermal energy density, $E_{0}$, and specific heat per unit volume, $C_{V}$ are evaluated as function of the Debye temperature, $\theta_{\mathrm{D}}$, using physical constants table [14].

TABLE I

$C_{i j}^{0}$ and $C_{i j k}^{0}$ in $\left[10^{11}\right.$ dyne $\left./ \mathrm{cm}^{2}\right]$ for Pt.

\begin{tabular}{c|c|c|c|c}
\hline \hline$C_{11}^{0}$ & $C_{12}^{0}$ & $C_{111}^{0}$ & $C_{112}^{0}$ & $C_{144}^{0}$ \\
\hline 19.14 & 6.57 & -308.04 & -26.62 & 10.63
\end{tabular}

Thermal relaxation time has been obtained using Eq. (4), taking thermal conductivity from physical constants table [15], Gruneisen parameter $\left\langle\gamma_{i}^{j}\right\rangle$, square average Gruneisen parameter $\left\langle\left(\gamma_{i}^{j}\right)^{2}\right\rangle$, and non-linearity constants $D_{l}$ and $D_{s}$ along different crystallographic directions are evaluated using Eq. (6) and $D_{l}$ and $D_{s}$ are given in Table II.

Sound absorption coefficients for longitudinal wave, $\left(\frac{\alpha}{f^{2}}\right)_{l}$ and for shear wave, $\left(\frac{\alpha}{f^{2}}\right)_{s}$ are evaluated using Eq. (5). Temperature variation of $\left(\frac{\alpha}{f^{2}}\right)_{l}$ and $\left(\frac{\alpha}{f^{2}}\right)_{s}$ along different crystallographic directions of propagation and polarization are shown in Figs. 1-3. Temperature variation of $\left(\frac{\alpha}{f^{2}}\right)_{\text {th }}$ along different directions for longitudinal wave has been presented in Table III.

Values of SOEC and TOEC at $0 \mathrm{~K}$ are given in Table I. Cagin et al. [2] have evaluated SOEC for $\mathrm{Pt}$ using computer simulation technique at different temperatures. Their values at $300 \mathrm{~K}$ are $C_{11}=28.96 \times 10^{11} \mathrm{dyne} / \mathrm{cm}^{2}, C_{12}=$ $23.93 \times 10^{11}$ dyne $/ \mathrm{cm}^{2}$, and $C_{44}=6.50 \times 10^{11} \mathrm{dyne} / \mathrm{cm}^{2}$, while our values at the same temperature are $C_{11}=19.19 \times 10^{11} \mathrm{dyne} / \mathrm{cm}^{2}, C_{12}=19.14 \times 10^{11} \mathrm{dyne} / \mathrm{cm}^{2}$ $C_{44}=6.51 \times 10^{11}$ dyne $/ \mathrm{cm}^{2}$. Thus there is good agreement between values of $C_{11}$ and $C_{44}$. Deviations in value of $C_{11}$ and slight deviation in value of $C_{44}$ can be attributed to the approach adopted by them. They have used NVE molecular dynamics simulation, in which they have not considered certain parameters. Also our approach is very simple, which involves only two basic parameters, nearest-neighbour distance and hardness parameter. 
TABLE II

Temperature variation of $D_{l}$ and $D_{s}$ along different directions for Pt.

\begin{tabular}{c|c|c|c|c|c|c|c|c}
\hline \hline \multirow{2}{*}{ Temp. [K] } & \multicolumn{2}{|c|}{$\langle 100\rangle$} & \multicolumn{4}{c|}{$\langle 110\rangle$} & \multicolumn{3}{c}{$\langle 111\rangle$} \\
\cline { 2 - 8 } & $D_{l}$ & $D_{s}$ & $D_{l}$ & $\begin{array}{c}D_{s}^{*} \\
\langle 001\rangle\end{array}$ & $\begin{array}{c}D_{s}^{* *} \\
\langle 1 \overline{1} 0\rangle\end{array}$ & $D_{l}$ & $\begin{array}{c}D_{s}^{+} \\
\langle\overline{1} 10\rangle\end{array}$ & $\begin{array}{c}D_{s}^{++} \\
\langle 11 \overline{2}\rangle\end{array}$ \\
\hline 100 & 28.32 & 4.08 & 38.67 & 17.11 & 54.87 & 79.73 & 28.27 & 22.59 \\
200 & 30.38 & 4.01 & 41.29 & 17.00 & 54.08 & 84.75 & 27.87 & 22.35 \\
300 & 30.73 & 3.94 & 41.43 & 16.88 & 53.26 & 86.68 & 27.46 & 22.11 \\
400 & 29.73 & 3.87 & 39.65 & 16.77 & 52.47 & 86.35 & 27.05 & 21.88 \\
500 & 29.04 & 3.81 & 38.33 & 16.66 & 51.70 & 86.38 & 26.67 & 21.66 \\
600 & 28.30 & 3.74 & 36.99 & 16.55 & 50.96 & 86.26 & 26.29 & 21.44 \\
700 & 27.57 & 3.68 & 35.68 & 16.45 & 50.24 & 86.09 & 25.93 & 21.30 \\
800 & 26.84 & 3.63 & 34.40 & 16.34 & 49.55 & 85.87 & 25.24 & 21.03 \\
900 & 26.16 & 3.57 & 33.21 & 16.25 & 48.87 & 85.65 & 25.24 & 20.84 \\
1000 & 25.48 & 3.52 & 32.03 & 16.16 & 48.22 & 85.40 & 24.92 & 20.65 \\
1100 & 24.05 & 3.47 & 30.96 & 16.07 & 47.59 & 85.18 & 24.60 & 20.48 \\
1200 & 24.22 & 3.42 & 29.90 & 15.98 & 46.98 & 84.93 & 24.30 & 20.30 \\
1300 & 23.65 & 3.38 & 28.93 & 15.89 & 46.39 & 84.71 & 24.00 & 20.14 \\
1400 & 23.08 & 3.34 & 28.00 & 15.81 & 45.81 & 84.49 & 23.74 & 19.98 \\
1500 & 22.54 & 3.29 & 27.11 & 15.73 & 42.25 & 84.28 & 23.44 & 19.82
\end{tabular}

Average and square average Gruneisen parameters have the values as expected [16]. Non-linearity constants ratio along all the three directions of propagation is as expected [16].

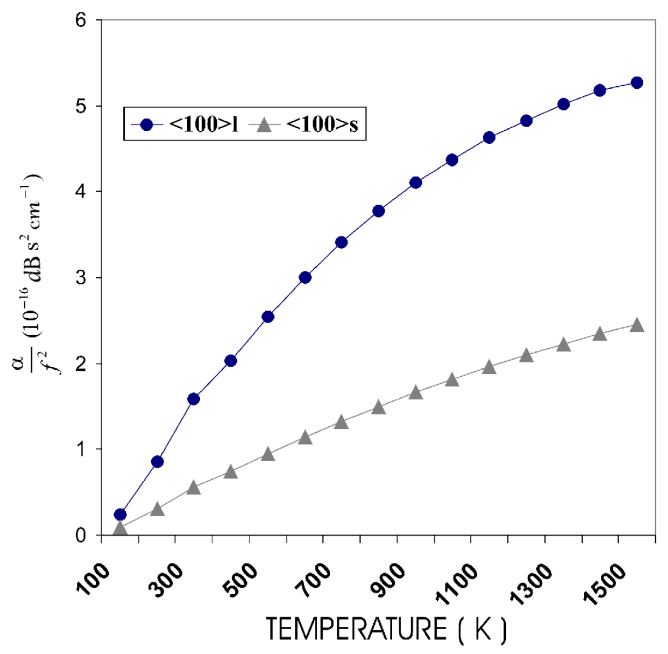

Fig. 1. Variation of $\left(\frac{\alpha}{f^{2}}\right)$ with temperature for longitudinal and shear waves along $\langle 100\rangle$ direction for platinum. 


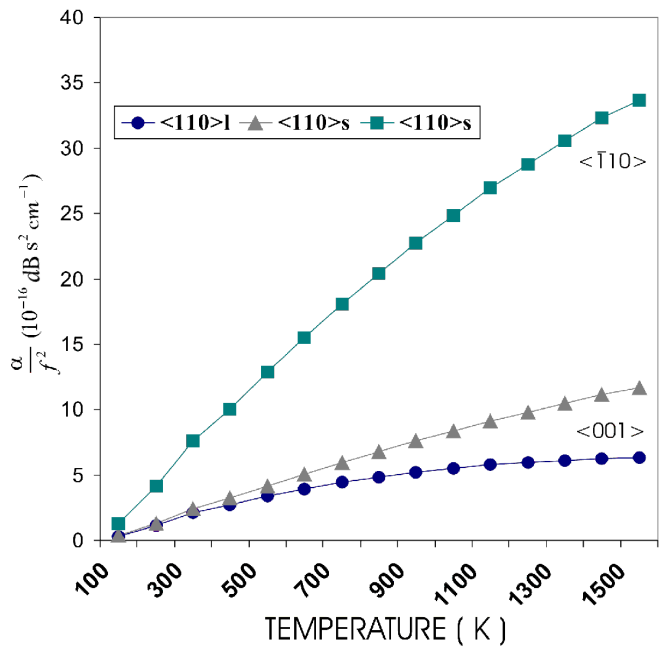

Fig. 2. Variation of $\left(\frac{\alpha}{f^{2}}\right)$ with temperature for longitudinal and shear waves along $\langle 110\rangle$ direction for platinum (polarisations are shown).

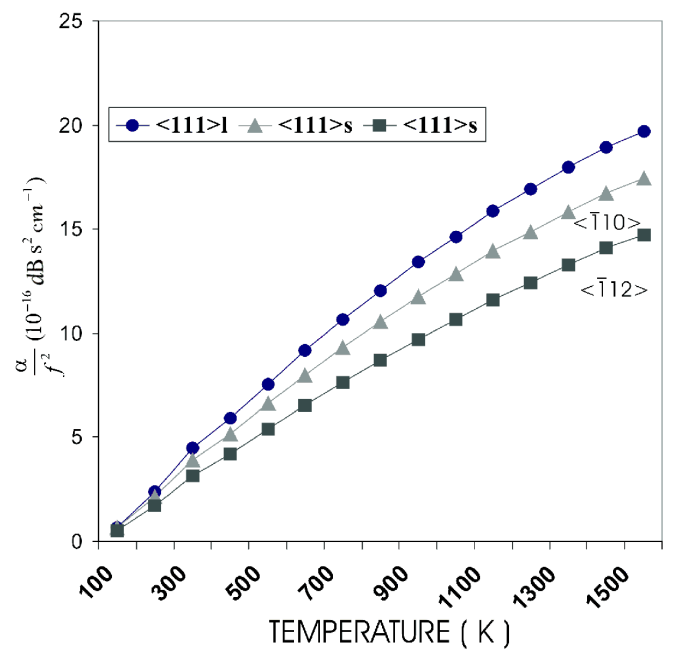

Fig. 3. Variation of $\left(\frac{\alpha}{f^{2}}\right)$ with temperature for longitudinal and shear waves along $\langle 111\rangle$ direction for platinum (polarisations are shown).

From Figs. $1-3$ it can be seen that temperature variation of $\left(\frac{\alpha}{f^{2}}\right)$ is similar along all the directions for longitudinal and shear waves. $\left(\frac{\alpha}{f^{2}}\right)_{l}$ and $\left(\frac{\alpha}{f^{2}}\right)_{s}$ increase with temperature. At lower temperatures rate of increase is large, as temperature increases beyond $500 \mathrm{~K}$, rate of increase becomes small. Beyond $500 \mathrm{~K}$ rate of variation of $\left(\frac{\alpha}{f^{2}}\right)$ with temperature is small. 
Rate of increase in $\left(\frac{\alpha}{f^{2}}\right)_{l}$ with temperature is little larger for longitudinal waves compared to shear waves. This may be attributed to larger value of thermal relaxation time, $\tau$ for longitudinal waves compared to that of shear waves (Eqs. (4) and (5)).

TABLE III

Parameter $\left(\frac{\alpha}{f^{2}}\right)_{\text {th }}$ in $\left[10^{-18} \mathrm{~dB} \mathrm{~s}^{2} \mathrm{~cm}^{-1}\right]$ at different temperature for $\mathrm{Pt}$.

\begin{tabular}{c|c|c|c}
\hline \hline \multirow{2}{*}{ Temp. $[\mathrm{K}]$} & \multicolumn{3}{|c}{ Direction of propagation } \\
\cline { 2 - 4 } & $\langle 100\rangle$ & $\langle 110\rangle$ & $\langle 111\rangle$ \\
\hline 100 & 0.98 & 0.55 & 1.65 \\
200 & 2.25 & 1.26 & 3.63 \\
300 & 3.36 & 1.85 & 5.13 \\
400 & 4.21 & 2.36 & 6.25 \\
500 & 5.00 & 2.78 & 7.08 \\
600 & 5.67 & 3.15 & 7.69 \\
700 & 6.25 & 3.47 & 8.13 \\
800 & 6.75 & 3.74 & 8.41 \\
900 & 7.18 & 3.97 & 8.57 \\
1000 & 7.54 & 4.16 & 8.62 \\
1100 & 7.83 & 4.32 & 8.60 \\
1200 & 8.07 & 4.45 & 8.51 \\
1300 & 8.27 & 4.55 & 8.36 \\
1400 & 8.44 & 4.62 & 8.19 \\
1500 & 8.51 & 4.67 & 7.92
\end{tabular}

From Table III and from the values of $\left(\frac{\alpha}{f^{2}}\right)_{\text {th }}$ given in Figs. $1-3$, it can be seen that $\left(\frac{\alpha}{f^{2}}\right)_{\text {th }}$ along all the directions is negligible in comparison to $\left(\frac{\alpha}{f^{2}}\right)_{l}$ or $\left(\frac{\alpha}{f^{2}}\right)_{s}$. Although experimental results are not available in the temperature range studied, yet on the basis of the above discussions it can be concluded that the present approach is correct.

\section{Acknowledgments}

One of us (R.K. Singh) gratefully acknowledges financial support F. No. 670/SPT/2002(MRP/NRCB) from University Grants Commission, Government of India. 


\section{References}

[1] D. Verma, M.L. Verma, A. Verma, Ind. J. Phys. A 78, 337 (2004).

[2] T. Cagin, G. Dereli, M. Uludogan, M. Tomak, Phys. Rev. B 59, 3648 (1999).

[3] S.K. Kor, R.K. Singh, Acta Phys. Pol. A 80, 805 (1991).

[4] R.K. Singh, J.P. Acad. Sci. 5, 80 (1996).

[5] S.K. Kor, R.K. Singh, J. Acoust. Soc. India 21, 203 (1993).

[6] S.K. Kor, R.K. Singh, Acustica 79, 83 (1993).

[7] S.K. Kor, R.K. Singh, Acustica 78, 292 (1993).

[8] R.R. Yadav, O.K. Pandey, Acta Phys. Pol. A 107, 933 (2005).

[9] K. Brugger, Phys. Rev. A 133, 1611 (1964).

[10] G. Leibfried, W. Ludwig, in: Solid State Physics, Eds. F. Seitz, D. Turnbull, Academic Press Inc., New York 1961.

[11] J. Fabian, P.B. Allen, Phys. Rev. Lett. 82, 1478 (1999).

[12] W.P. Mason, Physical Acoustics, Vol. III B, Academic Press, New York 1965.

[13] R.R. Yadav, D. Singh, Acoust. Phys. 49, 595 (2003).

[14] AIP Handbook, Academic Press, New York 1981.

[15] CRC Data Series, CRC Press, Florida 1999-2000.

[16] S.K. Kor, R.K. Yadav, Kailash, J. Phys. Soc. Japan 55, 282 (1986). 